\title{
Raman Mapping Coupled to Self Modelling MCR-ALS Analysis to Estimate Active Cosmetic Ingredient Penetration Profile in Skin
}

\author{
Aline Stella \\ Université de Tours \\ Franck Bonnier \\ Université Francois-Rabelais de Tours, franck.bonner@univ-tours.fr \\ Ali Tfayli \\ Universite Paris-Saclay
}

See next page for additional authors

Follow this and additional works at: https://arrow.tudublin.ie/nanolart

Part of the Chemistry Commons

\section{Recommended Citation}

Aline, S. et al (2020). Raman mapping coupled to self-modelling MCR-ALS analysis to estimate active cosmetic ingredient penetration profile in skin. Journal of BIOphotonics, vol.13(11). doi:10.1002/ jbio. 202000136 .

This Article is brought to you for free and open access by the NanoLab at ARROW@TU Dublin. It has been accepted for inclusion in Articles by an authorized administrator of ARROW@TU Dublin. For more information, please contact arrow.admin@tudublin.ie, aisling.coyne@tudublin.ie,gerard.connolly@tudublin.ie.

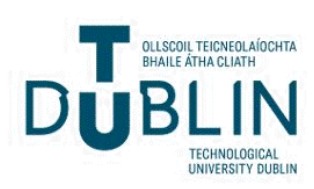




\section{Authors}

Aline Stella, Franck Bonnier, Ali Tfayli, Florent Yvergnaux, Hugh Byrne, Igor Chourpa, Emilie Munnier, and Clovis Tauber 


\title{
Raman mapping coupled to self modelling MCR-ALS analysis to estimate active cosmetic ingredient penetration profile in skin
}

\author{
Aline Stella ${ }^{1}$ Franck Bonnier ${ }^{2} \mid$ Ali Tfayli $^{3} \mid$ Florent Yvergnaux $^{4} \mid$ Hugh J Byrne $^{5} \mid$ Igor Chourpa $^{2} \mid$ Emilie Munnier $^{2}$ | \\ Clovis Tauber ${ }^{1 *}$
}

1 UMR U1253, iBrain, Université de Tours, Inserm, Tours, France.

2 EA6295 Nanomédicament et Nanosondes, Université de Tours, Tours, France.

3 U-Psud,Univ.Paris-Saclay, Lip(Sys) 2, EA7357, UFR-Pharmacy, ChatenayMalabry, France

4 Bioeurope Solabia Group, Anet, France 5 FOCAS Research Institute, TU Dublin, City Campus, Dublin 8, Ireland

\section{* Correspondence}

Clovis Tauber, UMR U1253 iBrain, Université de Tours, Inserm,

10 Boulevard Tonnellé, 37032 Tours, France

Email: clovis.tauber@univ-tours.fr

\begin{abstract}
Confocal Raman Mapping (CRM) is a powerful, label free, non-destructive tool, enabling molecular characterisation of human skin with applications in the dermocosmetic field. Coupling CRM to multivariate analysis can be used to monitor the penetration and permeation of Active Cosmetic Ingredients (ACI) after topical application. It is presently illustrated how Multivariate Curve Resolution Alternating Least Squares (MCR-ALS) can be applied to detect and semi-quantitatively describe the diffusion profile of Delipidol ${ }^{\circledR}$, a commercially available slimming ACI, from Raman spectral maps. Although the analysis outcome can be critically dependent on the a priori selection of the number of regression components, it is demonstrated that profiling of the kinetics of diffusion into the skin can be established with or without additionnal spectral equality constraints in the multivariate analysis, with similar results. Ultimately, MCR-ALS, applied without spectral equality contraints, specifically identifies the ACI as one of main spectral components enabling to investigate its distribution and penetration into the stratum corneum and underlying epidermis layers.
\end{abstract}

\section{KEYWORDS}

Confocal Raman Mapping, Multivariate Curve Resolution Alternating Least Squares, Delipidol ${ }^{\circledR}$, human skin, penetration profiles

\section{1 | INTRODUCTION}

The dense corneocyte layers and lipid rich composition of the Stratum Corneum (SC) of the skin ensure a particularly effective defence against exogenous agents. It is well accepted that the resulting barrier function can strongly compromise the efficiency of Active Cosmetic Ingredients (ACI) by limiting their penetration. However, many commercialised ACI in skin products have cellular targets located in deeper layers of the skin, below the SC. As a result, evaluation of cosmetic products, i.e. biological testing, to validate the diffusion of the ACI into the skin cannot be circumvented. While chromatographic techniques can be considered as gold standards [1], enabling determination of the kinetics of penetration with high accuracy and sensitivity, large amounts of solvents are used for such analysis, making the method non eco-friendly (an increasing concern in the cosmetic industry). Moreover, tape stripping protocols employed for chromatographic analysis lead to lack of accuracy at a sub-micrometric level, due the nature of the mechanical separation of cell layers with adhesive films. Over the last 30 years, Raman spectroscopy has gained popularity in this specific field of application [2]. This nondestructive, label free and reagent (solvent) free technique provides molecular fingerprints based on the intrinsic, inelastic scattering of light, when the sample is illuminated with a laser source without adding any tracer. Moreover, coupling the molecular specificity of the analysis with the micrometric spatial resolution offers a relevant platform for evaluation of local diffusion and distribution of active molecules [3]-[9].

However, bio-analytical applications of Raman spectroscopy, along with Near Infrared (NIR) and Fourier Transform Infrared (FT-IR) spectroscopy, are hampered by the complexity of the spectral signatures originating from combined contributions from all the chemical compounds in the analysed area. Therefore, advanced data mining protocols are usually necessary to extract and interpret the spectral information inside the large data sets generated by hyperspectral mapping, and pre-processing can also play a key role in correcting or diminishing spectral variability originating from several optical and instrumental effects [10] Recently, the development of multivariate analysis (i.e. chemometrics) has greatly contributed to increasing the potential of spectroscopy mapping for tissue, cell, and surface analysis of material but also in pharmaceutical and cosmetic fields [3], [7], [19], [20], [11]-[18]. Principal Components Analysis (PCA) and K-means clustering methods are examples of descriptive unsupervised methods, widely used to better understand the hyperspectral data [21]. The former, PCA, is often applied to reduce the number of variables in a multidimensional data set and the analysis of the identified principal components can provide some information about the origin of the variability of the data. The second approach, K-means clustering, is a method commonly employed in spatial mapping applications, by which regions of similar spectral profiles are associated. A number of examples of applications of PCA and/or K-means clustering to understand how skin components evolve under specific conditions [11], [12] and to detect the presence of exogenous molecules [7], [13] have been reported. Such 
methods are particularly interesting for interpretation of large hyperspectral maps containing hundreds to thousands of data points. However, they are unable to provide the spectral signatures of specific biochemical components and their respective abundance from data. Thus, to access the distribution of specific chemicals, it is necessary to use more elaborate unmixing methods. Decomposing each pixel of an image in a combination of estimated constituent spectra, according to their respective abundance, enables both qualitative and quantitative characterisation. Therefore, biomedical profiles of cells and tissues can be analysed to access subtle changes linked to biological mechanisms or pathological development [22], [23]. Among the available unmixing approaches, Classic Least Squares (CLS) and Independent Components Analysis (ICA) are methods commonly employed in a wide range of industrial applications, including monitoring the penetration of molecules such as penetration enhancers, vitamins or other active ingredients [3], [13]-[15], control of pharmaceutical products or detection of counterfeit medicines [16], [17]. While CLS uses a collection of spectral references of all components in the sample to estimate their respective abundance, ICA only requires an estimation of the number of components present to decompose them. Singular Value Decomposition (SVD) is widely used to estimate this parameter, while Durbin-Watson criterion and KMO index are also regularly used [24], [25]. However, since ICA does not impose a non-negativity constraint on the constituent spectra, the biochemical interpretation of spectral decomposition is more complicated.

A recent study has investigated the penetration of Delipidol ${ }^{\circledR}$ through the skin by means of Non-negative Constrained Least Squares (NCLS) applied to skin cross sections [26]. Reconstructed distribution maps and penetration profiles of this molecule were estimated using a measured pure spectrum of ACI. However, this method presents some limitations as its performance relies on the availability of a large number of spectra from control samples but also on the feasibility of collecting highly reliable reference spectra from pure compounds. The use of such a reference spectral data base excludes the possibility of, for example, band shifts due to molecular interactions.

In contrast, Multivariate Curve Resolution Alternating Least Squares (MCR-ALS) regression analysis is an unmixing method which can provide an accurate molecular decomposition of the spectroscopic information contained in the data set, while also dealing with such band shifts [27]. MCR-ALS has been applied to detect modification in ingredients and their dosing in falsified medicine [16], [18][20], [28]. The method has also been applied on FT-IR and Raman data to study effects of pathology, anatomy, environmental or genetic factors [27] and on Raman data collected from plant cell walls to study the mechanical stability of cells by changing their form, their thickness or their composition [29]. The advantages of MCR-ALS [30] over other multivariate approaches lies in the possibility to add some constraints to the model, such as non-negativity or equality constraints. The equality constraints can be a known spectrum or/and concentration, while retaining a reduced number of components. Additionally, the method does not require large control data sets. Therefore, MCR-ALS is an attractive alternative to most common methods proposed to evaluate the efficiency and innocuity of cosmetic products, starting with establishing their distribution profiles of the ACI to study their kinetics of penetration in skin layers.

This work aims to evaluate the MCR-ALS algorithm for the tracking of an ACI through the skin with Raman spectroscopy. The investigation addresses whether an a priori knowledge of the tracked molecule is necessary, and thus whether forcing one spectral signature to be that of the ACI signature changes the performance of the MCR-ALS approach in its data decomposition and fitting.

\section{2 | Experimental data set}

The present work proposes to revisit and optimise the data analysis of previously published material [26]. The initial work was performed using NCLS and full details are provided in reference. Briefly, Raman spectra were acquired using a LabRam (Horiba Jobin-Yvon, Villeneuve-d'Ascq, France). Samples of Punica granatum seed oil hydroxyphenethyl esters, commercialised as Delipidol ${ }^{\circledR}$, were kindly provided by BioEurope Solabia, France Solabia (Pantin, France). It is a patented formulation used as an anticellulite ACI. Ethanolic solution of 5\% Delipidol ${ }^{\circledR}$ has been topically applied for $1 \mathrm{~h}, 2 \mathrm{~h}$ and $3 \mathrm{~h}$ onto human reconstructed skin samples (Episkin ${ }^{\circledR}$, Lyon, France). Raman maps were collected from $20 \mu \mathrm{m}$ thick cross-sections prepared using a cryo-microtome and placed on Raman grade $\mathrm{CaF}_{2}$ substrates. As mentioned in [26], ethanol is a solvent which can act as penetration enhancer leading to superior diffusion of Delipidol $^{\circledR}$. However, the aim of the experiment was to construct a set of Raman images with noticeable variations in the ACI concentrations to illustrate the application of multivariate analysis, i.e MCR-ALS.

\section{3 | Data processing and analysis}

\section{1 | Data pre-processing}

All Raman spectra were subject to pre-processing, including spectral denoising, baseline correction, followed by normalisation. All pre-processing steps were performed in MATLAB (R2017b).

\subsection{1 | Spectral denoising}

The spectra were first filtered with a PCA to remove the noise [31]. PCA is a multivariate data analysis technique that aims to summarise and simplify the information available with as little information loss as possible. This simplification consists of transforming the variables to ones uncorrelated with each other. Once the uncorrelated principal components have been selected, all data are projected onto them, to go back to the original space. Hence, the noise is reduced. Note that, in the spectroscopy field, it is common to reduce the dimensionality of the spectral data set while preserving its main features to make it possible to remove the noise [32], 
[33]. In this work, $1 \%$ of the number of spectral data was used as the number of loadings for the data projection (more details in Supplementary Materials FIGURE S1).

\subsection{2 | Baseline Correction}

During spectral acquisition, instrumental phenomena, scattering or intrinsic fluorescence by certain molecules under the excitation laser can introduce a background to spectra. This baseline has to be corrected prior to data analysis.

The Rubberband method is a well-established and previously employed background correction algorithm [10], [34]-[37] that was preferred in this study.

The Rubberband method estimates a piecewise polynomial baseline. First, a set of supports points is determined, such that the region below these points form a convex hull. Then, a polynomial curve is estimated between each support point. The polynomial order was set to 1 to avoid overcorrection of spectra. Finally, the set of these estimated polynomials compose the baseline and they are subtracted from the spectra (FIGURE S2).

\subsection{3 | Normalisation}

The last step of the pre-processing corresponds to the data normalisation. In this work, it was achieved by calculating the ratio of spectra to their respective Euclidian norms.

\section{2 | Data analysis}

\subsection{1 | MCR-ALS}

Unmixing methods assume that the data matrix $D$, of size $N \times N_{\lambda}$ where $N$ and $N_{\lambda}$ are respectively the number of pixels/spectra and Raman shifts, can be expressed as a linear combination of pure constituent spectra, weighted by their respective abundance (FIGURE 1). Mathematically, the data $D$ are considered to be the product of a pure spectra matrix $S^{T}$, composed of $K$ components, multiplied by the matrix of relative concentration $C$ of these $K$ components in each pixel, as following:

$$
D=C S^{T}+E
$$

where $E$ is the residual matrix of the linear model (1).

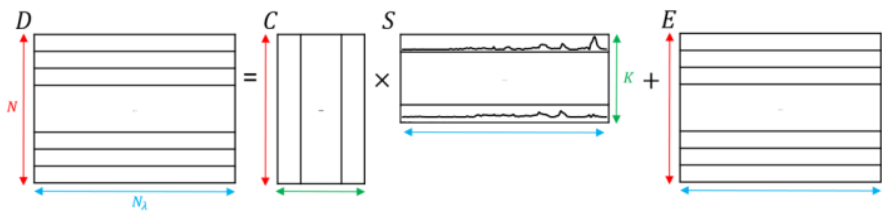

FIGURE 1 - Linear mixing model

Furthermore, the MCR-ALS algorithm is a self-modelling method. It resolves an optimisation problem through the minimisation of the adjustment errors $E$ of the linear model. The minimisation problem to be solved is expressed as:

$$
\min \left\|D-C S^{T}\right\|
$$

Some constraints have been added to this optimisation problem to respect some logical and chemical properties, such as positivity of the estimated Raman spectra and their relative concentrations. The non-negativity constraint on spectra and relative concentration is introduced to the minimisation problem, such that equation (1) becomes:

$$
\min _{S \geq 0, C \geq 0}\left\|D-C S^{T}\right\|^{2}
$$

The minimisation problem (4) can be solved by different algorithms. The MCR-ALS method is one of them and the algorithm is designed to better fit the model to the data by alternatively minimising residuals by least squares fitting, according to the spectral matrix $S$ and to the associated abundance matrix $C$. Hence, the ALS aspect of the implementation is expressed as:

$$
\begin{aligned}
& C=D S\left(S^{T} S\right)^{-1} \\
& S^{T}=\left(C^{T} C\right)^{-1} C^{T} D
\end{aligned}
$$

Ultimately, MCR-ALS relaxes the hypothesis of the presence of pure components within the data and it assumes that each pixel is a mixed signal (FIGURE 33).

Although the MCR-ALS algorithm aims at solving a global minimisation problem, its initialisation can influence the quality of the results. Therefore, it is preferable to use a method such as SIMPLISMA (SIMPLe-to-use-InteractiveSelf-Modelling-Mixture-Analysis) [25] to provide initial estimations of pure spectra, somehow close to the solution, as a starting point, rather than a random approach that could lead to unrealistic, outlier local minima [38].

SIMPLISMA estimates components of a mixture based on the purity of spectra, which is defined as a modified coefficient of variation:

$$
P_{i}=\frac{\sigma_{i}}{\mu_{i}+\beta}
$$

where $P_{i}$ is the purity coefficient for a given spectrum $S_{i}, \sigma_{i}$ the standard deviation, $\mu_{i}$ the mean spectrum and $\beta$ is a coefficient offset between $1 \%$ and $5 \%$ of the mean. The higher the coefficient, the more significant is the difference to the mean, and therefore the component spectrum is considered purer. Ultimately, the purest spectra are selected based on the purity coefficient and also on their orthogonality to each other.

Alternatively, if constituent spectra or/and relative concentrations are known, the MCR-ALS method allows the addition of constraints to impose this information onto the constructed model. It consists of including the known spectra into the spectral initialisation and setting the constraints on the spectra and relative abundances in the respective matrices $S$ and $C$ at each iteration. It should be noted, that, in the absence of calibration, concentrations $C$ of constituents are relative and their manifestation in the complex mixture is dependent on the relative strength of the Raman scattering cross sections, giving rise to the spectra $S$.

The MCR-ALS algorithm converges when the difference in the model adjustment between two iterations is smaller than a set threshold. MCR-ALS was applied using a multiset approach, i.e. all images corresponding to $1 \mathrm{~h}, 2 \mathrm{~h}$ and $3 \mathrm{~h}$ have 
been decomposed simultaneously to consider the kinetics of Delipidol $^{\circledR}$ penetration and increase the coherency of the component extracted to more specifically reconstruct semiquantitative distribution maps.

It should be noted that the application of added constraints such as non-negativity of spectra/abundances or correspondence of one component with a reference spectrum and the multiset approach promote singularity of the solution and reduces its rotational ambiguity [39]. The MCR-Bands algorithm described in [39] makes it possible to estimate the rotational ambiguity of a solution. It consists of calculating the relative signal contribution of every component of the maximum and minimum band boundaries of the feasible solutions and computes their differences. In the case where there is no rotational ambiguity, the estimated maximum and minimum band boundaries are close to the solution and the computed difference is close to 0 . In this work, MCR-ALS and MCR-Bands algorithms have been applied using the scripts from MCR-ALS GUI 2.0 software in Matlab [40].

\subsection{2 | Number of components}

Components are estimated Raman signatures used to decompose each pixel of the image. Therefore, it is necessary to set the number of components $K$ to be estimated in the MCR-ALS computation. The parameter $K$ plays a critical role in the decomposition of spectral maps, and the difficulty with biological samples lies in the fact that this number is unknown and difficult to estimate. Therefore, in order to reduce the subjectivity, different mathematical approaches are available to provide an estimation and guide the analysis. The most commonly used is SVD. Two other criteria, the Durbin-Watson criterion [25] and the Kaiser-Meyer-Olkin Index (KMO) [24] have also been recently proposed.

All these criteria have been applied to the evaluation of Raman maps of reconstructed skin samples exposed to Delipidol $^{\circledR}$. While SVD is determined directly from the data maps, the other two decision criteria are based on SIMPLISMA outputs using a number of constituents ranging from 1 to 20 . Further information can be found in supplementary data.

\subsection{3 | Analysis tools}

Some statistic tools are needed to analyse the data decomposition resulting from MCR-ALS. Correlation coefficients are used to quantitatively compare spectral components and highlight any possible matches to reference spectra, while histograms of experimental residuals are used to qualitatively evaluate the model fitting. They are described below:

- Correlation coefficient - The correlation coefficient measures the linear link between several variables. Two components are positively and negatively correlated if their correlation coefficient is respectively close to 1 and -1 . Note that a null value does not imply independence. Moreover, the squared correlation coefficient, known as coefficient of determination, corresponds to the percentage of variations in the spectral values of a component that can explain the variations of the spectral values of another one. In other words, this squared value corresponds to the recovery rate of spectral bands between two components. In general, a correlation coefficient superior to 0.9 in absolute value is statistically considered as very high correlation, and therefore such a correlation coefficient is considered to be satisfactory, since more than $80 \%$ of variations of spectral values will be interrelated [41].

- Histogram of residuals - A histogram is a graphical representation to visualise the data set repartition. All ranges of values are binned in classes, categorised according to the number of values included in them. In other words, for this work, all Raman intensities of mean model residuals are organised in a series of intervals and the number of Raman shifts having a Raman intensity included in each of them is associated to them. The histogram shape provides some information about the value distribution; in particular, a Gaussian shape means that the residual noise is Gaussian.

\section{4 | RESULTS AND DISCUSSION}

\subsection{Spectral characterisation}

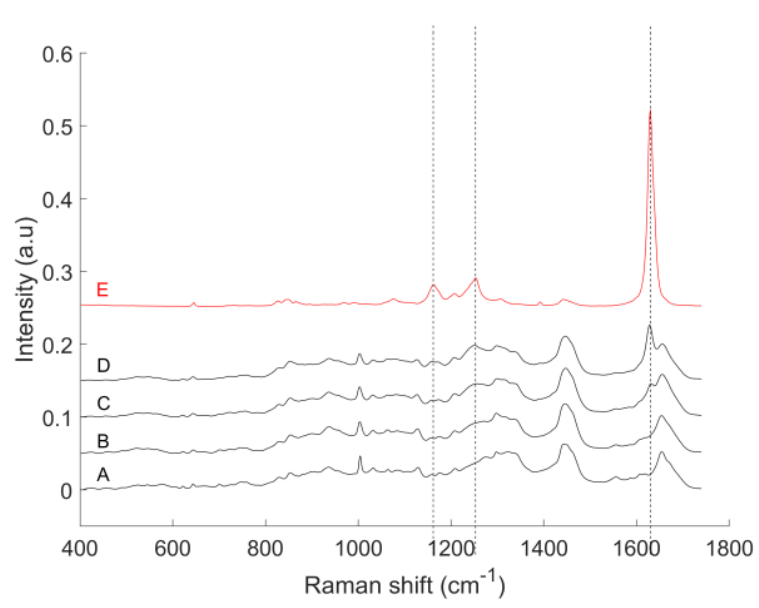

FIGURE 2 - Comparison of data mean spectrum at each exposure time to the ACI with the reference spectrum of the ACI. A: control; B: $1 \mathrm{~h} ; \mathbf{C}: 2 \mathrm{~h} ; \mathbf{D}: 3 \mathrm{~h}$ and $\mathbf{E}$ : pure spectrum of Delipidol ${ }^{\circledR}$. Data are pre-processed with the Rubberband baseline.

Delipidol $^{\circledR}$ has a characteristic Raman signature, with a combination of intense specific peaks easily discernible and weaker spectral bands partially overlapping with skin features (FIGURE 2-E). As such, it is an interesting candidate to evaluate unmixing approaches such as MCRALS. Spectral characterisation of Delipidol ${ }^{\circledR}$ is presented in FIGURE 2-E, showing dominant spectral features at 1630 $\mathrm{cm}^{-1}\left(\mathrm{C}=\mathrm{C}\right.$ stretching of the aromatic ring), $1254 \mathrm{~cm}^{-1}$ $(\mathrm{C}-\mathrm{C}-\mathrm{C})$ and $1162 \mathrm{~cm}^{-1}$. Other weaker bands at $1441 \mathrm{~cm}^{-1}$, $1393 \mathrm{~cm}^{-1}, 1307 \mathrm{~cm}^{-1}, 1251 \mathrm{~cm}^{-1}, 1207 \mathrm{~cm}^{-1}, 1076 \mathrm{~cm}^{-1}, 991$ $\mathrm{cm}^{-1}, 969 \mathrm{~cm}^{-1}, 865 \mathrm{~cm}^{-1}, 843 \mathrm{~cm}^{-1}$ and $827 \mathrm{~cm}^{-1}$ can also be observed. 
Mean spectra of Raman maps of control, $1 \mathrm{~h}, 2 \mathrm{~h}$ and $3 \mathrm{~h}$ exposure time are also illustrated in FIGURE 2 as an overall initial comparison of spectra. For illustration purposes, mean spectra presented have been obtained using the preprocessing strategy described above. In addition to those of Delipidol ${ }^{\circledR}$, Raman spectra collected from skin samples exhibit a complex signature originating from combined spectral features of physiological constituents. The amide I band located at $1655 \mathrm{~cm}^{-1}$ indicates the skin is predominantly composed of proteins such as keratin and other cellular proteins [11]. Content may vary between the upper layers corresponding to SC and other layers containing higher proliferating cell density (e.g. basal layer). The position and shape of the amide I band reflects changes in protein composition but also in their conformation (secondary structure) [42], and therefore can substantially vary across the skin tissue section. Similarly, spectra contain a wealth of information about lipids, contributing peaks identified at $1064 \mathrm{~cm}^{-1}, 1129 \mathrm{~cm}^{-1}$ and $1297 \mathrm{~cm}^{-1}$ but also bands of DNA and nucleic acids observed at $783 \mathrm{~cm}^{-1}(\mathrm{O}-\mathrm{P}-\mathrm{O}), 813 \mathrm{~cm}^{-1}$ (O-P-O), $1086 \mathrm{~cm}^{-1}(\mathrm{O}-\mathrm{P}-\mathrm{O}), 1252 \mathrm{~cm}^{-1}$ (guanine, cytosine),
$1324 \mathrm{~cm}^{-1}$ (DNA) and $1340 \mathrm{~cm}^{-1}$ (DNA) [42]. However, Delipidol $^{\circledR}$ exhibits a characteristic Raman signature, with a combination of intense specific peaks and weaker spectral bands (FIGURE 2-E), which are easily discernible superimposed on the spectrum of skin (FIGURE 2$\mathbf{A}, \mathbf{B}, \mathbf{C}, \mathbf{D})$ even in the mean spectra of the skin cross-section, in which its localised contribution is naturally diluted. As a result, each pixel of the image can be considered a complex spectral mixture corresponding to the local biochemical composition tempered with contributions from the ACI. MCR-ALS applied to Raman maps is expected to deliver more specific information about its distribution in the different layers of the epidermis and consequently enable construction of penetration profiles.

\section{2 -MCR-ALS applied to ACI penetration study using $K=4$ components with non-negativity constraint only}

This section presents results of MCR-ALS applied only with non-negativity constraints on spectra and relative

\section{No spectral equality constraint}

\section{Spectral equality constraint on the $\mathrm{ACl}$}

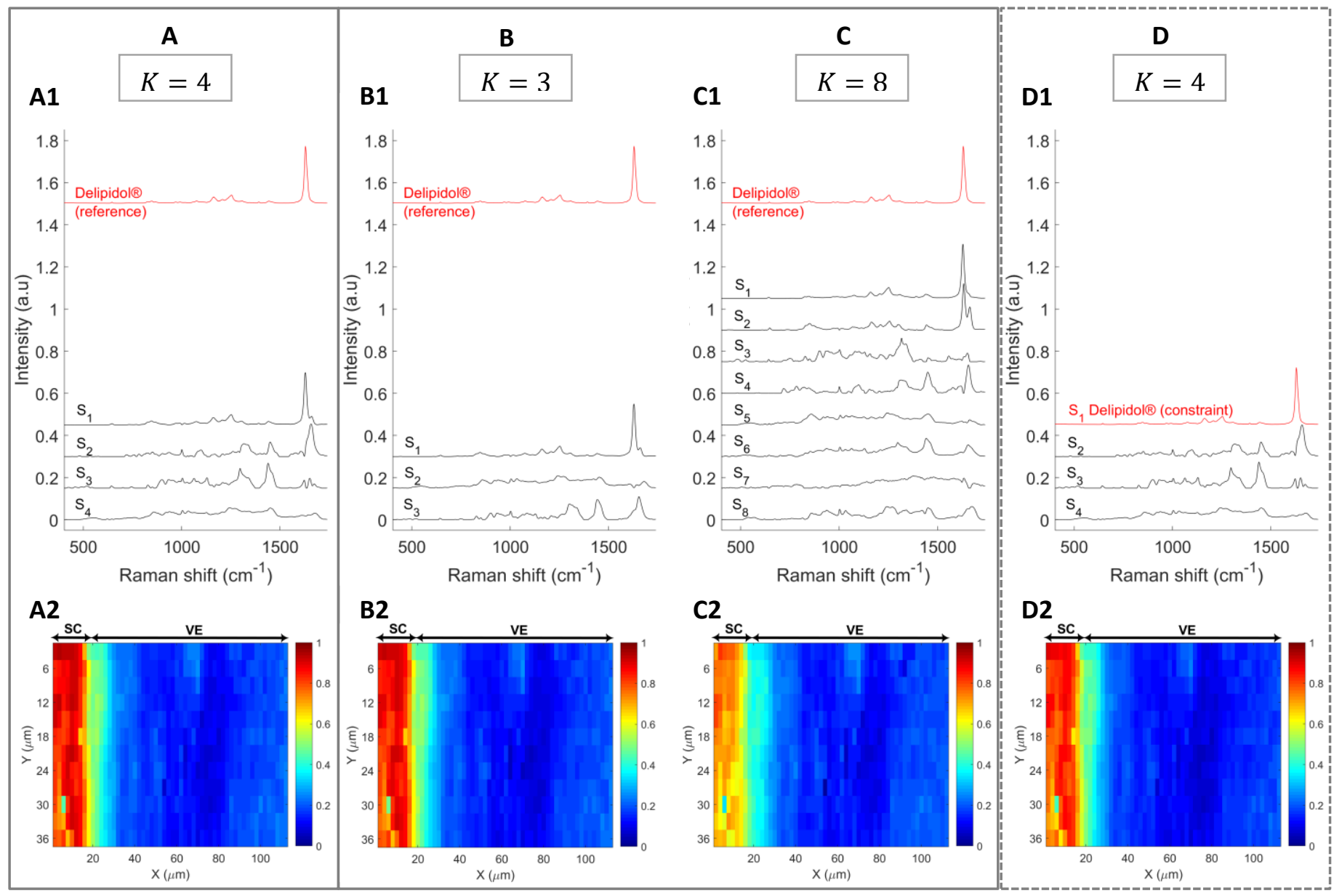

FIGURE 3 - MCR-ALS applied with only non-negativity constraints for $K=4(\mathbf{A})$ and $K=\{3,8\}(\mathbf{B}, \mathbf{C})$ and with an additional spectral equality constraint on the ACI for $K=4$ (D) on pre-processed data; A1-B1-C1-D1: Spectra estimated with $K$ components initialized by SIMPLISMA (offset for clarity); A2-B2-C2-D2: Reconstructed maps of Delipidol ${ }^{\circledR}$ in skin samples at $3 \mathrm{~h}$ exposure time. Maps obtained using estimating spectrum of ACI $S_{1}(\mathbf{A} 2-\mathbf{B} 2-\mathbf{C} 2)$ or referential spectrum $S_{1}(\mathbf{D 2})$. 

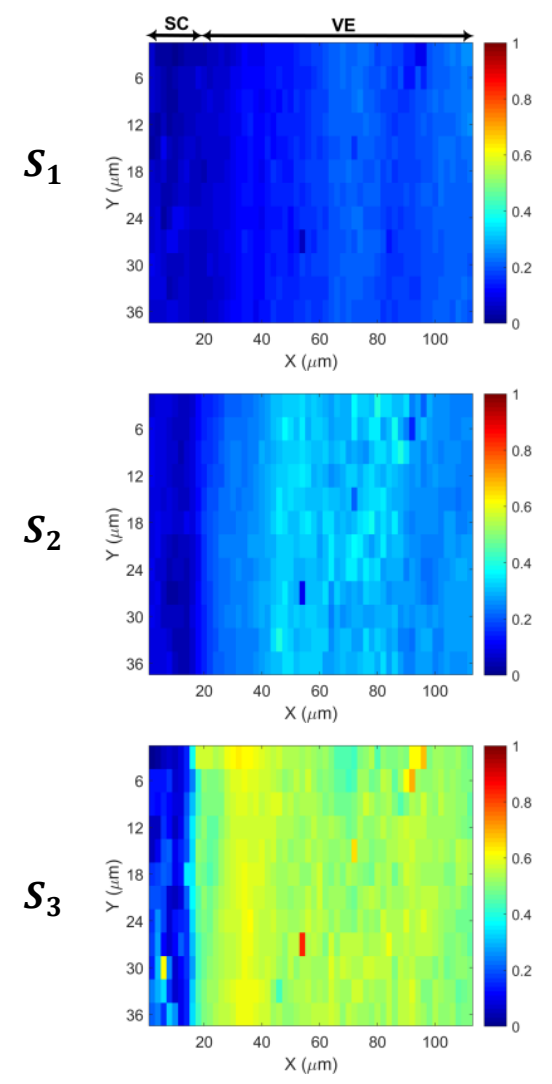

FIGURE 4 - Reconstructed concentration distribution maps of $S_{1}$, $S_{2}$ and $S_{3}$ in skin samples at $3 \mathrm{~h}$ exposure time. They resulted from MCR-ALS applied only with non-negativity constraints applied with $K=4$.

concentrations and no ACI reference to guide the algorithm, using $K=4$ components. The 4 estimated spectral components are displayed in FIGURE 3-A, with corresponding reconstructed distribution map for Delipidol ${ }^{\circledR}$. Results are illustrated with data subjected to Rubberband baseline correction, derived from a multiset analysis, including maps from $1 \mathrm{~h}, 2 \mathrm{~h}$ and $3 \mathrm{~h}$.

Spectral components are identified as $S_{i}$ for $i=1, \ldots, 4 \in \mathbb{N}$ (FIGURE 3-A1) and their display order has been organised so that they are more easily readable and comparable. Compared to the reference spectrum of Delipidol ${ }^{\circledR}$, spectral component $S_{1}$ exhibits strong similarities. Although a shoulder at $1661 \mathrm{~cm}^{-1}$ suggests that a small residual feature from the amide I band remains, the MCR-ALS applied with only non-negativity constraints to spectra and abundances was able to identify a spectral component with strong similarities to the pure spectrum of the ACI studied (correlation coefficient of 0.9746), highlighting that the reference spectrum is not necessary to construct accurate distribution maps. Distribution maps for components $S_{2}, S_{3}$ and $S_{4}$ obtained with $K=4$ are displayed in FIGURE 4. These components exhibit features related to biochemical constituents of the skin, such as lipids, proteins and nucleic acids [11], consistent with those observed in the mean spectra of FIGURE 2. Although it is not the subject of the current study, the combination of Raman/MCR-ALS can be further employed to study the skin composition, and any changes to it as a result of permeation of the ACI.

Moreover, the value of the differences of the relative contributions of the 4 components of maximum and minimum band boundaries estimated by application of the MCR-Bands algorithm are respectively $1.16 \times 10^{-3}$, $8.00 \times 10^{-3},-5.81 \times 10^{-3}$ and $-25.61 \times 10^{-3}$ for $S_{1}, S_{2}$, $S_{3}$ and $S_{4}$, indicating that the rotational ambiguities are negligible.

For each exposure time, penetration profiles of the ACI were averaged over all samples (black curves in FIGURE 5) displaying the permeation of Delipidol ${ }^{\circledR}$ into the SC for the 1h experiment, followed by progressive diffusion into deeper layers of the epidermis after $2 \mathrm{~h}$ and $3 \mathrm{~h}$ exposure with a concomitant increase in the outer layer $(0-20 \mu \mathrm{m})$. It was observed that average penetration profiles calculated using the MCR-ALS applied with only non-negativity constraints were close to the profiles obtained with a more constrained approach such as NCLS [26] (FIGURE 5).

\subsection{Comparison of numbers of $K$ parameters}

The choice of $K=4$ in section 4.2.2 was rather subjective and based on the similarity of $S_{1}$ with the pure spectrum of Delipidol $^{\circledR}$. However, the number of parameters chosen can have an impact on the model performance.

It was anticipated that selecting a number of components for the MCR-ALS applied to biological samples would be a difficult task. Despite the existence of criteria to support the analyst in this decision, the observation of SVD, DWC and KMOI all suggested that a high number of components would be necessary to encompass the entire biochemical spectral variability (FIGURE S4). However, the purpose of using advanced unmixing methods is to provide simplified information about the distribution of Delipidol ${ }^{\circledR}$ which is easily interpretable. Moreover, the criteria described took into account the global information contained in the spectral maps and they did not necessarily directly reflect the optimal number of components to accurately and specifically detect and localise the ACI onto chemical maps.

Plots obtained using SVD, DWC and KMOI are provided in FIGURE S4. There is no unambiguous decision about the optimal number of components to use for the spectra decomposition. While SVD suggests 11 components would be necessary to reach $95 \%$ of explained variance, DWC and KMO tends to indicate that numbers between 4 and 8 could capture the relevant spectral information contained in Raman maps. Here are presented results from different sizes of the spectral base $(K=\{3,8\})$ to better understand the impact of the parameter $K$ on the spectral unmixing and the model fitting to the data. Hence, in order to evaluate the reliability of MCR-ALS, the correlation coefficients have been computed to estimate similarities between spectral components and the reference spectrum of Delipidol ${ }^{\circledR}$, and the interpretation of experimental residuals distribution by means of histograms has been explored. 


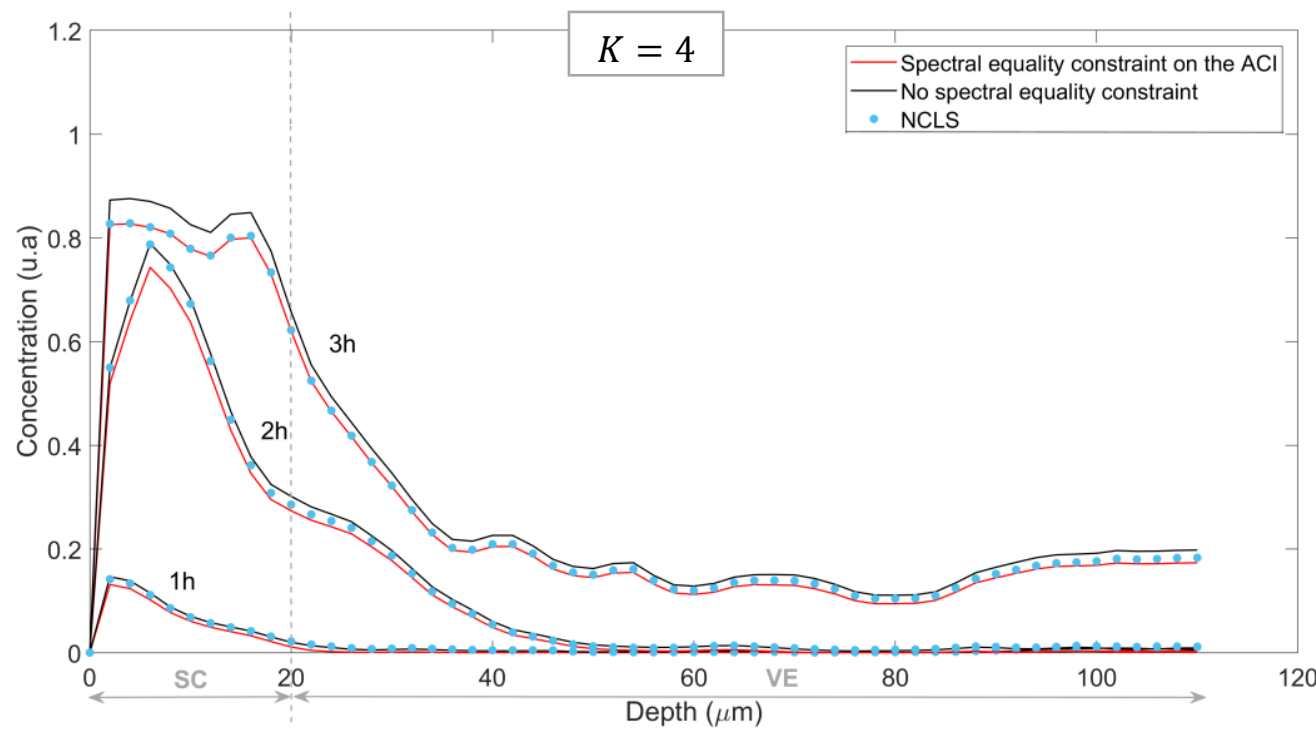

FIGURE 5 - Average penetration profiles of the Delipidol ${ }^{\circledR}$ for $1 \mathrm{~h}, 2 \mathrm{~h}$ and $3 \mathrm{~h}$ of exposure time obtained by MCR-ALS applied with (red) versus without (black) spectral equality constraints on the ACI and using $\mathrm{K}=4$. The average concentrations obtained by NCLS are illustrated by blue points.

TABLE 1 - Correlation coefficients between estimated spectra $S_{i=1, \ldots, K}$ obtained by MCR-ALS applied with and without spectral equality constraints on the ACI $(K=\{3,4\})$ from data corrected with Rubberband method.

\begin{tabular}{|c|c|c|c|c|c|c|c|}
\hline \multirow{2}{*}{$\begin{array}{l}\text { No spectral } \\
\text { equality constraint }\end{array}$} & \multicolumn{3}{|c|}{$K=\mathbf{3}$} & \multicolumn{4}{|c|}{$K=4$} \\
\hline & $\begin{array}{c}S_{1} \\
\text { Delipidol }^{\circledR}\end{array}$ & $S_{2}$ & $S_{3}$ & $\begin{array}{c}S_{1} \\
\text { Delipidol }^{\circledR}\end{array}$ & $S_{2}$ & $S_{3}$ & $S_{4}$ \\
\hline$S_{1}$ & 0.9753 & 0.0512 & 0.3452 & 0.9746 & 0.3218 & 0.1393 & 0.1821 \\
\hline$S_{2}$ & 0.0054 & 0.9994 & 0.4070 & 0.2518 & 0.9983 & 0.4214 & 0.3911 \\
\hline$S_{3}$ & 0.2664 & 0.4425 & 0.9971 & 0.0681 & 0.4535 & 0.9979 & 0.7216 \\
\hline$S_{4}$ & & & & 0.1110 & 0.4206 & 0.6790 & 0.9999 \\
\hline
\end{tabular}

TABLE 2 - Mean concentration of estimated ACI after $1 \mathrm{~h}, 2 \mathrm{~h}$ and $3 \mathrm{~h}$ exposure time obtained by MCR-ALS applied with and without spectral equality constraints on the ACI for $K=\{3,4\}$ from data corrected with Rubberband method.

\begin{tabular}{|c|c|ccc|ccc|}
\multicolumn{2}{|c|}{} & \multicolumn{3}{|c|}{$\boldsymbol{K = 3}$} & \multicolumn{3}{c|}{$\boldsymbol{K}=\mathbf{4}$} \\
\cline { 2 - 8 } \multicolumn{2}{|c|}{} & $\mathbf{1 h}$ & $\mathbf{2 h}$ & $\mathbf{3 h}$ & $\mathbf{1 h}$ & $\mathbf{2 h}$ & $\mathbf{3 h}$ \\
\hline \multirow{2}{*}{$\begin{array}{c}\text { No spectral } \\
\text { equality } \\
\text { constraint }\end{array}$} & $\mathbf{0}-\mathbf{2 0} \boldsymbol{\mu m}$ & 0.0635 & 0.5439 & $\mathbf{0 . 8 0 5 0}$ & 0.0691 & 0.5364 & $\mathbf{0 . 8 0 9 8}$ \\
\hline $\begin{array}{c}\text { Spectral } \\
\text { equality } \\
\text { constraint on } \\
\text { the ACI }\end{array}$ & $\mathbf{4 0 - 1 1 0 \mu m}$ & 0.0012 & 0.1737 & 0.3477 & 0.0067 & 0.1753 & 0.3498 \\
\hline
\end{tabular}


Spectral components $S_{i}$ for $i=1, \ldots, K \in \mathbb{N}$ with $K=$ $\{3,8\}$ used for the MCR-ALS applied with only nonnegativity constraints are displayed in FIGURE 3-B1 and FIGURE 3-C1, with corresponding reconstructed distribution maps for Delipidol ${ }^{\circledR}$ in FIGURE 3-B2 and FIGURE 3-C2. While distribution maps for $3 \mathrm{~h}$ exposure time appeared similar for $K=3$ and $K=4$, the outcome with $K=8$ diverged for the first 2 models with clearly an significantly lower abundance of Delipidol ${ }^{\circledR}$ in the $20 \mu \mathrm{m}$, corresponding to the SC. Observations of spectral components highlighted that, with $K=8$, features of Delipidol ${ }^{\circledR}$ at $1630 \mathrm{~cm}^{-1}, 1442 \mathrm{~cm}^{-1}, 1254 \mathrm{~cm}^{-1}$ and $1162 \mathrm{~cm}^{-}$ ${ }^{1}$ have been also found in $S_{2}$ along with other skin features. It is an example of over decomposition of the spectral component of interest. While increasing the number of $K$ parameters should deliver overall a better fitting of the model, presently the Delipidol ${ }^{\circledR}$ is no longer considered as single pure component thus no interpretation of its distribution can be made.

In comparison $S_{1}$ components respectively calculated with $K=3$ and $K=4$ appears quite comparable. To estimate the degree of similarity correlation coefficients of estimated spectral components using $K=\{3,4\}$ are presented in TABLE 1 (table including $K=8$ is provided in
Supplementary Materials - TABLE S1). To be noted that $S_{1}$ Delipidol $^{\circledR}$ refers to the pure spectrum of the ACI. It is observed that the correlation coefficients between the spectral component of Delipidol ${ }^{\circledR}$ estimated from the algorithm using only the non-negativity constraints and the reference pure spectrum used $\left(S_{1}\right.$ Delipidol $\left.^{\circledR}\right)$ are really high, with respective values of $0.9753,0.9746$ for $K=3$ and $K=$ 4. Moreover, the estimated spectral component $S_{2}$ and $S_{3}$ for $K=3$ and $K=4$ do not match with the reference ACI spectrum (correlation coefficients are respectively 0.0054 , 0.2664 and $0.2518,0.0681$ ), suggesting the component $S_{1}$ can be considered close to spectroscopically pure. In contrast, $S_{2}$ for $K=8$ has a relatively large correlation coefficient of 0.8801 with the signature of Delipidol ${ }^{\circledR}$ (Supplementary Materials - TABLE S1). As mentioned above, this is a result of over-decomposition of the spectral signatures resulting with presence of Delipidol ${ }^{\circledR}$ features in multiple spectral components.

The study investigates the applicability of MCR-ALS to construct distribution maps of the ACI as function of time. It is expected that a gradient will be observed from the surface of the skin $(S C)$ towards deeper layers of the viable epidermis (VE). Therefore, the comparison criteria between models relies on the abundance of Delipidol ${ }^{\circledR}$ according to depth.

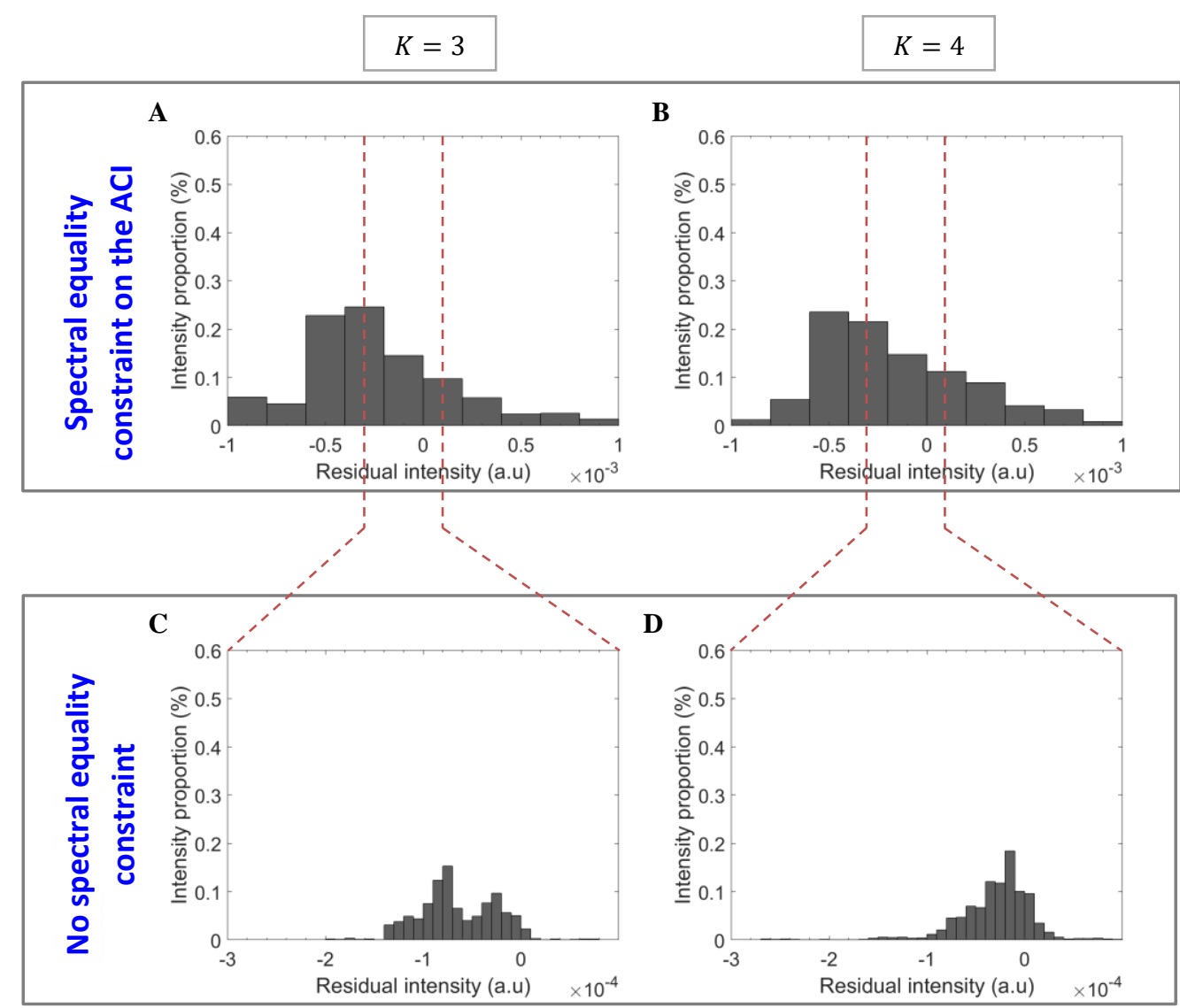

FIGURE 6 - Histograms of mean residuals obtained by MCR-ALS applied with (A-B) and without (C-D) spectral equality constraints on the ACI for $K=\{3,4\}$ from data preprocessed with Rubberband baseline correction. 
Here, numerical values of the relative mean concentrations for different depth ranges $0-20 \mu \mathrm{m}, 20-40 \mu \mathrm{m}$ and $40-110 \mu \mathrm{m}$ for $K=\{3,4\}$ have been calculated and are presented in TABLE 2 (top part for analysis using only non-negativity constraints). These values are also available for $K=8$ in Supplementary Materials - TABLE S2. For $K=3$ and $K=$ 4 sensibly identical results are obtained for $1 \mathrm{~h}, 2 \mathrm{~h}$ and $3 \mathrm{~h}$. For instance, after $3 \mathrm{~h}$ of exposure, relative mean respective concentrations are 0.8050 and 0.8098 in the $0-20 \mu \mathrm{m}$ range. Delipidol $^{\circledR}$ reached inferior layers of the epidermis progressively $(40-110 \mu \mathrm{m})$ and remained very concentrated on the upper skin layer $(0-20 \mu \mathrm{m})$ after $3 \mathrm{~h}$.

FIGURE 6-C, D presents the distributions of model residuals obtained by MCR-ALS using only non-negativity constraints for $K=\{3,4\}$. The analysis of residuals can bring additional insights about the impact of the number of components $K$ used. While for $K=4$ residuals display a nearly normal Gaussian distribution, which can correspond with the remaining noise, the shape of the distribution of residuals for $K=3$ is composed of two Gaussian, which tends to indicate that not all spectral components have been extracted from the data. The right-hand Gaussian part of the distribution can be interpreted as residual noise, whereas the left Gaussian indicates that some data variability is mathematically not modelled. Increasing to $K=4$ restores a unimodal Gaussian distribution, suggesting that no other significant spectral components remain in the residuals. Moreover, it is noted that the Gaussian distribution of the residuals shifts to more centered values when $K$ is increased, with a central tendency shifting from approximately $-0.85 \times 10^{-4}$ to $-0.25 \times 10^{-4}$ and finally $0.05 \times 10^{-4}$, for respectively $K=3, K=4$ and $K=8$. It suggests there is a mathematical overexpression of some data variability in the estimated model when $K$ is too small.

MCR-ALS enables extraction of the spectrum of ACI from the data set collected from the skin samples. Selecting too few components leads to an unsatisfactory model in terms of fitting errors. While the correlation coefficients (TABLE 1) and relative mean concentrations (TABLE 2) indicate that $K=3$ and $K=4$ deliver sensibly similar results, the experimental residuals suggest optimal fitting is obtained with 4 components. Ultimately, $K=4$ is a good trade-off between an acceptable fitting error and a coherent spectral decomposition.

\section{4 | Comparison of MCR-ALS applied with and without spectral equality constraints on the ACI}

Applying spectroscopic techniques for the analysis of penetration and/or permeation profiles of ACIs in the cosmetics field generally means the products themselves are available, and that pure, or reference, spectra can be collected before application onto the skin. In terms of application of multivariate analysis protocols, these reference spectra can be either employed to guide the analysis, by using them as spectral equality constraints, or to validate the analysis. In the following, the reference spectrum of Delipidol ${ }^{\circledR}$ is used as a spectral equality constraint on the ACI to help MCRALS to estimate its relative concentrations.

As mentioned in [27], when comparing methods with and without spectral equality constraints, it should be kept in mind that inserting more constraints in the MCR-ALS algorithm will naturally generally lead to a higher lack of fit due to a set of conditions to be respected that diminishes the flexibility of the model to find the optimal solution. Indeed, as MCR-ALS adjusts the fitting of spectral components through an iterative algorithm, using a fixed reference spectrum of a component leads to fewer degrees of freedom than an approach using only the non-negativity constraints. This should be considered when analysing the residuals. Adding some spectral equality constraints on the ACI can, however, in some situations, reduce overfitting to spectra that MCR-ALS would estimate as pure. but that could actually have a mixed character. For the current application, MCR-ALS was applied with $K=4$ components and with a spectral equality constraint on the first component of the model, $S_{1}$, fixed as a measured pure spectrum of Delipidol ${ }^{\circledR}$, while components $S_{2}, S_{3}$ and $S_{4}$ were calculated by the algorithm. As expected, adding a spectral equality constraint on $S_{1}$ leads to higher residuals (FIGURE 6). The analysis using a spectral equality constraint on the ACI indicates distributions closer to normal, but the fitting error is tenfold higher $\left(10^{-3}\right)$ compared to that not including it $\left(10^{-4}\right)$. The lack of fit [27], [40] was also calculated. The estimated model obtained by MCR-ALS applied without any spectral equality constraint had a lack of fit of $5.21 \%$. As expected, adding a spectral equality constraint on the ACI in the analysis increased this lack of fit by $2.17 \%$.

Nevertheless, the estimated spectral components and the reconstructed distribution maps for Delipidol ${ }^{\circledR}$ displayed in FIGURE 3-D do not exhibit any noticeable difference compared to the ones obtained by MCR-ALS applied without spectral equality constraint (FIGURE 3-A). Indeed, correlation coefficients for $S_{2}, S_{3}$ and $S_{4}$ between approaches using spectral equality constraint on the ACI or not were close to 1 , with respective values of $0.9983,0.9979$ and 0.9999 (TABLE 1). The spectral components estimated by applying a spectral equality constraint or not in the MCRALS analysis were found to be similar, for this application. This is understandable, as the component $S_{l}$ from the MCRALS applied without spectral equality constraint on ACI is really close to the reference spectrum of Delipidol ${ }^{\circledR}$, and thus the mathematical decomposition for other components leads to similar outcomes. This is confirmed by the trends in penetration profiles which are comparable for both approaches (FIGURE 5). Both average penetration profiles present the same higher accumulation of Delipidol ${ }^{\circledR}$ in the $S C$ and progressive decrease in deeper layers (TABLE 2). Note also that the kinetics of penetration described in this paper are close to the ones previously obtained with an NCLS approach [26] (FIGURE 5). However, comparison of relative concentrations highlights a slightly lower estimated abundance for the analysis using an additional spectral equality constraint on the ACI. For example, in the $S C(0-20$ $\mu \mathrm{m})$, when only non-negativity is applied to spectra and relative concentrations, estimated abundances were 0.0691 
(1h), $0.5364(2 \mathrm{~h})$ and $0.8098(3 \mathrm{~h})$, while when a spectral equality constraint on the ACI is added to the analysis, values of $0.0586,0.4995$ and 0.7638 were found. Despite this slight difference of relative abundances, the similarity of kinetics of penetration for both approaches support the possibility of using an MCR-ALS without spectral equality constraint on the ACI for our application.

In the field of cosmetic research and development, Raman spectroscopy can be a powerful tool to confirm a molecule is able to diffuse trough the main barrier function of the $S C$ and reach deeper cellular targets, without the requirement to access absolute concentrations. Images are a highly relevant means of representation of results with high impact in the industrial sector, especially in marketing, enabling comparative studies between different conditions, which is essential to support for example optimisation of formulations for skin care products. Presently, for the case of Delipidol ${ }^{\circledR}$, both approaches applied with and without spectral equality constraint on ACI enable to reach converging results as reconstructed images convey the same information (FIGURE 2-A,B,C,D) and it may be considered that the method addresses current needs for improved capabilities of Raman spectroscopy for skin analysis.

Previously NCLS was explored to probe the distribution of the ACI, rather than the specific skin components [26]. The approach remain to model as closely as possible the underlying skin contribution in spectra to more specifically extract Delipidol ${ }^{\circledR}$ relative concentrations. MCR-ALS, both approaches applying spectral equality constraint or not on the ACI, appears easier to employ, due to the low requirement for reference spectra to perform the analysis. In comparison, methods such as NCLS are strongly limited by their dependence on the degree of resemblance between the pure spectra and the constituents found in the skin, making the analysis less adaptive and thus prone to fitting errors.

MCR-ALS appears as potentially powerful alternative for unmixing of spectral component and investigation of the distribution of an ACI within skin cross sections. The approach using a spectral equality constraint on the ACI is quite relevant when a priori knowledge of the compound of interest is available. Adding a constraint of fixing one of the components with its pure spectrum enables to profile its distribution within Raman maps and reduces the rotational ambiguity of the results [39]. For the case of Delipidol ${ }^{\circledR}$, it is demonstrated that, despite a slight loss in the fitting quality, the advantage lies in the absence of ambiguity in the results.

However, the use of a spectral equality constraint in MCRALS could also suffer from limitations in the case where the ACI spectrum is changed because of, for example, the influence of the local environment as it progresses through the layers of the skin, crystallisation, accumulation in reservoirs, or metabolisation, or indeed when the target of the active is the molecular constituents of the skin itself. In such cases, the outcome of analysis constrained or not in spectral equality can be expected to diverge, and optimised protocols for the more flexible MCR-ALS without supplementary constraint could be more suited.

\section{5 | CONCLUSION}

Multivariate analysis provides promising unmixing methods to understand and track the kinetics of diffusion of ACI in skin samples from Raman data. In this work, results confirm that Raman microspectroscopy coupled with MCR-ALS is a powerful tool to detect and monitor an ACI penetration in the upper layers of the skin. It is observed that for the case of Delipidol ${ }^{\circledR}$ an a priori knowledge on the spectrum of ACI was not necessary for the analysis when using the MCR-ALS approach. The method applied without spectral equality constraint on the ACI was able to estimate a characteristic spectrum very close to that of ACI.

Ultimately, MCR-ALS decomposition provides similar penetration profile estimations to the previously tested NCLS, with the great advantage that it is a self modelling approach that does not require complex and sometimes unreliable large reference database of spectra from pure components or an extended number of control data set collected from control maps. In other words, the MCR-ALS coupled to Raman mapping appears to be an efficient tool to analyse and evaluate the effectiveness of ACI penetration and could support further transfer of the technique into the cosmetics industry.

\section{ACKNOWLEDGEMENTS}

This work is part of the MISTIC project (2017 - 00117681). We thank Conseil Régional Centre Val de Loire, ARD-2020 Cosmétosciences program and FEDER for financial support.

\section{REFERENCES}

[1] European Medicines Agency, "Draft Guideline on quality and equivalence of topical products," Comm. Med. Prod. Hum. Use, vol. 44, no. October, pp. 1-36, 2018.

[2] L. Franzen and M. Windbergs, "Applications of Raman spectroscopy in skin research - From skin physiology and diagnosis up to risk assessment and dermal drug delivery," Adv. Drug Deliv. Rev., vol. 89, pp. 91-104, 2015.

[3] M. Essendoubi, C. Gobinet, R. Reynaud, J. F. Angiboust, M. Manfait, and O. Piot, "Human skin penetration of hyaluronic acid of different molecular weights as probed by Raman spectroscopy," pp. 55-62, 2016.

[4] A. Tfayli, O. Piot, F. Pitre, and M. Manfait, "Follow-up of drug permeation through excised human skin with confocal Raman microspectroscopy," Eur. Biophys. J., vol. 36, no. 8, pp. 10491058, 2007.

[5] L. Binder et al., "Penetration monitoring of drugs and additives by ATR-FTIR spectroscopy/tape stripping and confocal Raman spectroscopy - A comparative study," Eur. J. Pharm. Biopharm., 
vol. 130, no. July, pp. 214-223, 2018.

[6] D. Lunter and R. Daniels, “Confocal Raman microscopic investigation of the effectiveness of penetration enhancers for procaine delivery to the skin," J. Biomed. Opt., vol. 19, no. 12, p. 126015, 2014.

[7] S. Mujica Ascencio et al., "Confocal Raman microscopy and multivariate statistical analysis for determination of different penetration abilities of caffeine and propylene glycol applied simultaneously in a mixture on porcine skin ex vivo," Eur. J. Pharm. Biopharm., vol. 104, pp. 51-58, 2016.

[8] G. J. Puppels, C. Nico, T. C. Bakker-Schut, J. de Sterke, and P. J. Caspers, "Skin penetration of topically applied products: quantitative in vivo analysis by Raman spectroscopy," in Proc. SPIE 11236, Biomedical Vibrational Spectroscopy 2020 : Advances in Research and Industry, 2020.

[9] P. J. Caspers et al., "Method to quantify the in vivo skin penetration of topically applied materials based on confocal Raman spectroscopy ," Transl. Biophotonics, vol. 1, no. 1-2, pp. 1-10, 2019.

[10] H. J. Byrne, P. Knief, M. E. Keating, and F. Bonnier, "Spectral pre and post processing for infrared and Raman spectroscopy of biological tissues and cells," Chem. Soc. Rev., vol. 45, no. 7, pp. 1865-1878, 2016.

[11] S. M. Ali et al., "Raman spectroscopic mapping for the analysis of solar radiation induced skin damage," Analyst, vol. 138, no. 14, pp. 3946-3956, 2013.

[12] F. J. González et al., "Noninvasive estimation of chronological and photoinduced skin damage using Raman spectroscopy and principal component analysis," Ski. Res. Technol., vol. 18, no. 4, pp. 442-446, 2012.

[13] V. K. Tippavajhala, T. de Oliveira Mendes, and A. A. Martin, "In Vivo Human Skin Penetration Study of Sunscreens by Confocal Raman Spectroscopy," AAPS PharmSciTech, vol. 19, no. 2, pp. 753-760, 2017.

[14] L. dos Santos et al., "Evaluation of penetration process into young and elderly skin using confocal Raman spectroscopy," Vib. Spectrosc., vol. 100, no. November 2018, pp. 123-130, 2019.

[15] F. D. Fleischli, S. Mathes, and C. Adlhart, "Vibrational Spectroscopy Label free non-invasive imaging of topically applied actives in reconstructed human epidermis by confocal Raman spectroscopy," Vib. Spectrosc., vol. 68, pp. 29-33, 2013.

[16] P.-A. Bonnet, C. Ghyselinck, C. Brenier, M. Martin, and H Rebiere, "Raman chemical imaging for spectroscopic screening and direct quantification of falsified drugs," J. Pharm. Biomed. Anal., vol. 148, pp. 316-323, 2017.

[17] M. Boiret, D. N. Rutledge, N. Gorretta, Y. M. Ginot, and J. M. Roger, "Application of independent component analysis on Raman images of a pharmaceutical drug product: Pure spectra determination and spatial distribution of constituents," J. Pharm. Biomed. Anal., vol. 90, pp. 78-84, 2014.

[18] L. Coic et al., "Comparison of hyperspectral imaging techniques for the elucidation of falsified medicines composition," Talanta, vol. 198, no. February 2019, pp. 457-463, 2019.

[19] B. Vajna, G. Patyi, Z. Nagy, A. Bódis, A. Farkas, and G. Marosi, "Comparison of chemometric methods in the analysis of pharmaceuticals with hyperspectral Raman imaging," J. Raman Spectrosc., vol. 42, no. 11, pp. 1977-1986, 2011

[20] S. J. Mazivila and A. C. Olivieri, "Chemometrics coupled to vibrational spectroscopy and spectroscopic imaging for the analysis of solid-phase pharmaceutical products: A brief review on non-destructive analytical methods," TrAC - Trends Anal. Chem., vol. 108, pp. 74-87, 2018.

[21] C. Krafft, G. Steiner, C. Beleites, and R. Salzer, "Disease recognition by infrared and Raman spectroscopy," Journal of Biophotonics, vol. 2, no. 1-2. pp. 13-28, 2009.

[22] K. W. Short, S. Carpenter, J. P. Freyer, and J. R. Mourant, "Raman spectroscopy detects biochemical changes due to proliferation in mammalian cell cultures," Biophys. J., vol. 88, no. 6, pp. 4274-4288, 2005

[23] I. R. Ramos et al., "Raman spectroscopy for cytopathology of exfoliated cervical cells," Faraday Discuss., vol. 187, pp. 187198,2016

[24] A. Kassouf, D. Jouan-Rimbaud Bouveresse, and D. N. Rutledge, "Determination of the optimal number of components in independent components analysis," Talanta, vol. 179, no. November 2017, pp. 538-545, 2018.

[25] S. Gourvénec, D. L. Massart, and D. N. Rutledge, "Determination of the number of components during mixture analysis using the Durbin-Watson criterion in the Orthogonal Projection Approach and in the SIMPLe-to-use Interactive Self-modelling Mixture Analysis approach," Chemom. Intell. Lab. Syst., vol. 61, no. 1-2, pp. 51-61, 2002.

[26] L. Miloudi et al., "Confocal Raman spectroscopic imaging for in vitro monitoring of active ingredient penetration and distribution in reconstructed human epidermis model," J. Biophotonics, vol. 11, no. 4, p. e201700221, Apr. 2018.

[27] J. Felten, H. Hall, J. Jaumot, R. Tauler, A. De Juan, and A. Gorzsás, "Vibrational spectroscopic image analysis of biological material using multivariate curve resolution-alternating least squares (MCR-ALS)," Nat. Protoc., 2015.

[28] D. N. Rutledge, E. de Paula, R. J. Poppi, H. Mitsutake, S. R. Castro, and M. C. Breitkreitz, "Comparison of different chemometric methods to extract chemical and physical information from Raman images of homogeneous and heterogeneous semi-solid pharmaceutical formulations," Int. J. Pharm., vol. 552, no. 1-2, pp. 119-129, 2018.

[29] B. Prats-Mateu, M. Felhofer, A. de Juan, and N. Gierlinger, 
"Multivariate unmixing approaches on Raman images of plant cell walls: New insights or overinterpretation of results?," Plant Methods, vol. 14, no. 1, pp. 1-20, 2018.

[30] A. De Juan, J. Jaumot, and R. Tauler, "Multivariate Curve Resolution (MCR). Solving the mixture analysis problem," Anal. Methods, vol. 6, no. 14, pp. 4964-4976, 2014.

[31] P. Koziol et al., "Comparison of spectral and spatial denoising techniques in the context of High Definition FT-IR imaging hyperspectral data," Sci. Rep., vol. 8, no. 1, pp. 1-11, 2018

[32] J. M. Shaver, "Chemometrics for Raman Spectroscopy," in Handbook of Raman spectroscopy. From the research laboratory to the process line., I. R. Lewis and H. G. M. Edwards, Eds. New York: Marcel Dekker, Inc., 2001, pp. 292-298.

[33] H. Martens and T. Naes, Multivariate Calibration. Chichester, UK: John Wiley and Sons, 1989.

[34] M. Pirzer and J. Sawatzki, "Method and Device for correcting a spectrum," 2006

[35] S. Wartewig, C. Schorn, and P. Bigler, "IR and Raman Spectroscopy: Fundamental Processing," Spectrosc. Tech., pp. 77-80, 2003.

[36] D. R. Parachalil, B. Brankin, J. Mclntyre, and H. J. Byrne, “Raman spectroscopic analysis of high molecular weight proteins in solution - considerations for sample analysis and data preprocessing," Analyst, vol. 143, no. 24, pp. 5987-5998, 2018.

[37] J. T. Bulmer, D. E. Irish, F. W. Grossman, G. Herriot, M. Tseng, and A. J. Weerheim, "Baseline Modeling With a Computerized Raman System.," Appl. Spectrosc., vol. 29, no. 6, pp. 506-511, 1975.

[38] W. Windig, J. Guilment, W. Winding, and J. Guilment, "Interactive self-modeling mixture analysis," Anal. Chem., vol. 63, no. 14, pp. 1425-1432, 1991.

[39] J. Jaumot and R. Tauler, "MCR-BANDS: A user friendly MATLAB program for the evaluation of rotation ambiguities in Multivariate Curve Resolution," Chemom. Intell. Lab. Syst., vol. 103, no. 2, pp. 96-107, Oct. 2010.

[40] J. Jaumot, A. de Juan, and R. Tauler, "MCR-ALS GUI 2.0: New features and applications," Chemom. Intell. Lab. Syst., vol. 140, pp. 1-12, 2015.

[41] R. Taylor, "Interpretation of the Correlation Coefficient: A Basic Review," J. Diagnostic Med. Sonogr., vol. 6, no. 1, pp. 35-39, 1990.

[42] S. M. Ali et al., "A comparison of Raman, FTIR and ATR-FTIR micro spectroscopy for imaging human skin tissue sections," Anal. Methods, vol. 5, no. 9, pp. 2281-2291, 2013.
Additional Supporting Information may be found online in the supporting information tab for this article. 